\title{
Actualities in the phytochemical research on selected terpenes
}

\author{
IGOR JERKOVIĆ ${ }^{*}$ \\ ŽELJAN MALEŠ² \\ MAJA FRIŠČIĆ ${ }^{2}$ \\ ${ }^{1}$ University of Split \\ Faculty of Chemistry and Technology \\ HR-21000 Split, Croatia \\ ${ }^{2}$ University of Zagreb \\ Faculty of Pharmacy and Biochemistry \\ HR-10000 Zagreb, Croatia
}

Accepted February 14, 2019

Published March 22, 2019

\begin{abstract}
A short review of our recent research on the essential oil phytochemical composition of Petasites albus (L.) Gaertn. and Petasites hybridus (L.) G. Gaertn., B. Mey. \& Scherb. (Asteraceae) as well as on the oils of Globularia cordifolia L., Globularia meridionalis (Podp.) O. Schwarz and Globularia punctata Lapeyr. (Plantaginaceae) is presented. All essential oils contained a variety of oxygenated sesquiterpenes among their major constituents, including a bakkane type sesquiterpene fukinanolid (bakkenolide A). The paper is focused on: i) a short overview of the abundance of major terpenes in the essential oils of Petasites and Globularia species from Croatia; ii) possible biosynthetic pathways of major identified sesquiterpenes; and iii) biological activities (literature data) of major sesquiterpenes from Petasites and Globularia species.
\end{abstract}

Keywords: Petasites species, Globularia species, oxygenated sesquiterpenes, bisabola-2,10-diene-1-one, fukinanolid (bakkenolide A), dehydrofukinone

Terpenes are derived biosynthetically from isopentenyl pyrophosphate (IPP) and dimethylallyl pyrophosphate (DMAPP) units. IPP and DMAPP can be produced through the 3-hydroxy-3-methylglutaryl coenzyme A (HMG-CoA) reductase pathway, known as the mevalonate pathway (discovered in the 1950s), via mevalonic acid as intermediate (1). Another pathway of producing terpenes is the 2-C-methyl-D-erythritol 4-phosphate/1-deoxyD-xylulose 5-phosphate pathway (MEP/DOXP pathway), which was discovered in the late 1980s $(1,2)$. IPP units are further connected to form precursors of each subclass of terpenes (geranyl pyrophosphate (GPP) for monoterpenes, farnesyl pyrophosphate (FPP) for sesquiterpenes, and others) that finally produce terpenes after initiation, propagation and termination sequences (1). Among terpenes, compounds with different biological activities (e.g., antioxidant, antimicrobial, cytotoxic, phototoxic, nuclear and cytoplasmic mutagenic, carcinogenic, antimutagenic properties, and others) can be found (3). Great chemical diversity of terpenes exists in nature and monoterpenes and sesquiterpenes are present in essential oils of the different aromatic plants $(1,4)$. They are isolated (4) by hydrodistillation (HD), steam distillation (SD), simultaneous distillation-extraction (SDE), solvent extraction (SE), ultrasonic solvent extraction (USE), solid-phase extraction (SPE), supercritical fluid extrac-

\footnotetext{
*Correspondence, e-mail: igor@ktf-split.hr
} 
tion (SFE), microwave-assisted extraction (MAE), stir-bar extraction (SBE), and different headspace (HS) techniques (dynamic and static). Phytochemical composition of essential oils has been ubiquitously determined by gas chromatography and mass spectrometry (GC-MS).

Our recent research $(5,6)$ was focused on the essential oil phytochemical composition of Petasites albus (L.) Gaertn. and P. hybridus (L.) G. Gaertn., B. Mey. \& Scherb. (Asteraceae) as well as on the oils of Globularia cordifolia L., G. meridionalis (Podp.) O. Schwarz and G. punctata Lapeyr. (Plantaginaceae) from Croatia. All the obtained essential oils were very complex, containing a variety of sesquiterpenes and characteristic oxygenated compounds were found among them, e.g., fukinanolid (bakkenolide A). The present paper is focused on: i) a short overview of the abundance of major terpenes in Petasites and Globularia species; ii) possible biosynthetic pathways of identified major sesquiterpenes; and iii) selected biological activities (literature data) of identified major sesquiterpenes from Petasites and Globularia species.

\section{Targeted sesquiterpenes}

Sesquiterpenes, as the largest group of terpenes, are subdivisible according to the arrangement of carbon atoms in the molecular skeleton $(4,7)$. This paper is focused on our recent research on the subgroups having their carbon atoms arranged in a monocyclic and bicyclic framework corresponding to bakkane, eremophilane and cadinane type sesquiterpenes found in the essential oils of two Petasites and three Globularia species. The obtained oils exhibited complex chemical composition, containing oxygenated sesquiterpenes. In contrast to our previous papers $(5,6)$, the novelty presented in this short review concerns a short presentation of their possible biosynthetic pathways and their observed biological activities from the literature.

\section{Major volatiles from $\mathrm{P}$. albus}

The major compound in the essential oil from P. albus leaves obtained by hydrodistillation (5) was a bisabolene type sesquiterpene bisabola-2,10-diene-1-one. In general, bisabola-2,10-diene-1-one is rarely found in essential oils. It is likely that it is derived (Fig. 1) from the FPP precursor generating $(E, E)$-farnesyl allylic cation, leading to nerolidyl pyrophosphate (NPP; 1, 4). NPP is another precursor that can undergo different types of reaction, but in this case, bisabolene synthase directs a single cyclization by electrophilic attack of C-1 onto the double bond of C-6 to form a six-membered ring. Tertiary bisabolyl cation yields different bisabolene derivatives by propagation (e.g., rearrangements, cyclizations) and termination reactions (e.g., proton eliminations, oxidations) including bisabola-2,10diene-1-one, $t$-muurolol and $t$-cadinol.

A bakkane type sesquiterpene fukinanolid (bakkenolide A) was another abundant compound in the essential oil of $P$. albus leaves along with two eremophilanes, $t$-muurolol and $t$-cadinol (5). The last two compounds were previously identified as biologically active molecules. Among others, $t$-cadinol exhibited a concentration-dependent smooth muscle relaxing effect on the isolated guinea pig ileum and a dose-dependent inhibitory effect on cholera toxin-induced intestinal hypersecretion in mice (8). $t$-Muurolol showed antifungal activity against Rhizoctonia solani and Fusarium oxysporum (9). If those two eremophilanes 


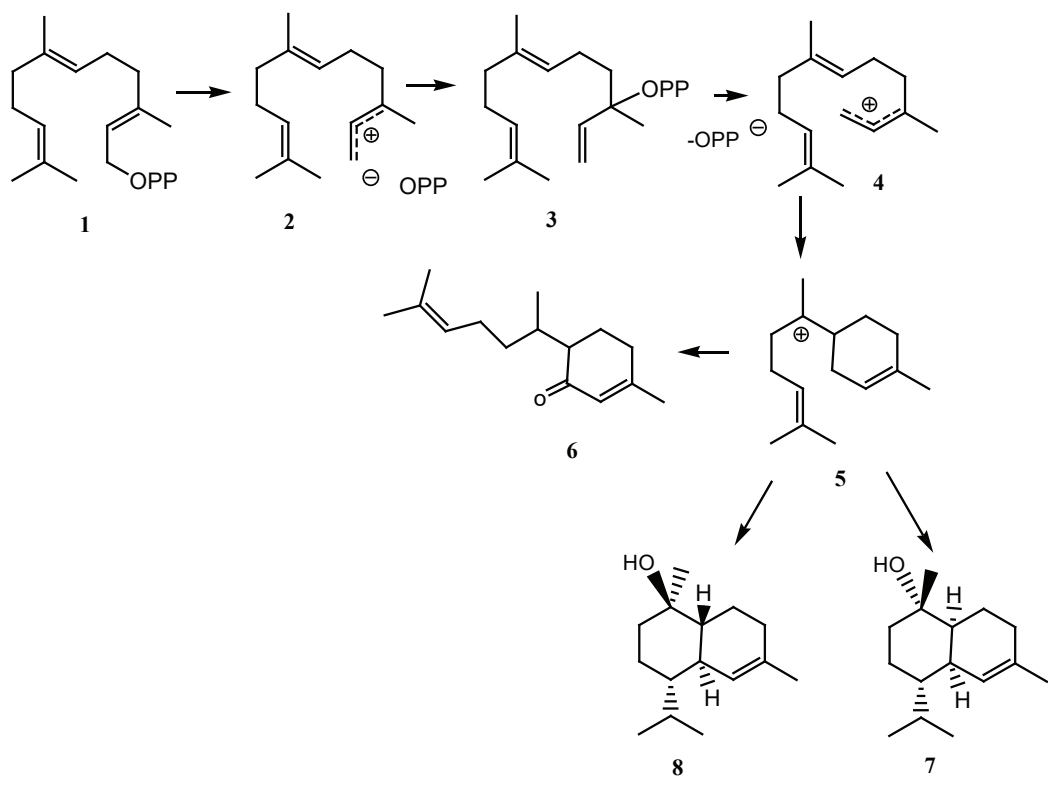

Fig. 1. Possible formation of bisabola-2,10-diene-1-one, $t$-muurolol and $t$-cadinol from farnesyl pyrophosphate (FPP): $\mathbf{1}$ - farnesyl pyrophosphate (FPP), $\mathbf{2}$ - (E,E)-farnesyl cation, $\mathbf{3}$ - nerolidyl pyrophosphate (NPP), 4 - nerolidyl cation, 5 - bisabolyl cation, $\mathbf{6}$ - bisabola-2,10-diene-1-one, 7 - $t$-muurolol, $8-t$-cadinol.

were in cis-configuration ( $t$-cadinol) or trans-configuration (t-muurolol) with axial hydroxyl at C-9, their antimite activities against Dermatophagoides pteronyssinus and D. farinae were lower than those of $\alpha$-cadinol (10). In the essential oil of $P$. albus leaves, germacrane sesquiterpenes germacrene D-4-ol and germacrene D were less abundant and so was the cadinane sesquiterpene $\delta$-cadinene (5). Bakkenolide A was the major component of $P$. albus flower stems and rhizome essential oils, while bisabola-2,10-diene-1-one and an undetermined furanoeremophilane-type sesquiterpene followed by other constituents were also present abundantly (5). Bakkenolide A was the main sesquiterpene lactone of P. albus (11, 12) and was found in much higher abundance in the buds, scales and flower stems in comparison with the leaves (11). Eremophilene, furanoeremophilane, several other eremophilene-type sesquiterpenes (albopetasin, petasalbin, albopetasol) and sesquiterpene hydrocarbons (e.g. petasitene, $\beta$-elemene, and humulene) were previously found in the rhizomes of $P$. albus from North Bohemia (13).

\section{Biosynthesis of bakkenolide A}

Bakkenolide $\mathrm{A}$ is a member of a large group of sesquiterpene butyrolactones. It is a tricyclic hydrindane with A and B rings fused in cis mode (Fig. 2). It also contains a spiro lactone moiety ( $\mathrm{C}$ ring) with an exocyclic methylene group. There are four chiral centers, present at C-4, C-5, C-7 (the spiro center), and C-10, which makes the synthesis more challenging (14). Bakkenolide $\mathrm{A}$ is presumed to be biogenetically related to eremophilanes 

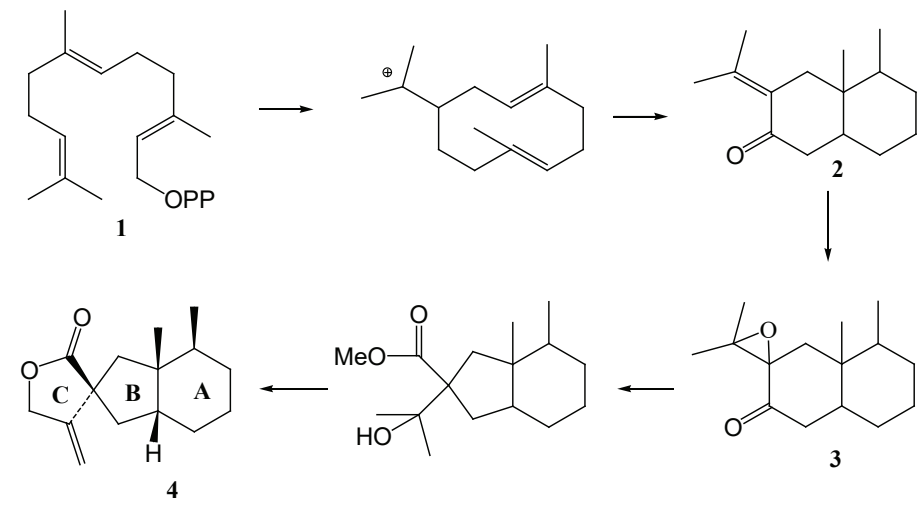

Fig. 2. Biosynthesis of fukinanolid (bakkenolide A): $\mathbf{1}$ - farnesyl pyrophosphate (FPP), $\mathbf{2}$ - fukinone, 3 - fukinone epoxide, 4 - bakkenolide A.

based on their frequent co-occurrence in nature and efficient "biomimetic" conversion of fukinone (15) to bakkenolide A. Biosynthesis of bakkenolide A is generally taken (16) to proceed as illustrated in Fig. 2.

Biosynthesis of bakkenolide A starts from FPP and proceeds through the cyclic cation intermediate, which undergoes oxidation and skeletal rearrangement to afford eremophilane fukinone (Fig. 2), a sesquiterpene ketone first isolated in 1968 from P. japonicus Maxim. (17). It has been proposed that fukinone is likely to be oxidized to fukinone epoxide and to subsequently undergo Favorskii skeletal rearrangement. Dehydration and further oxidation furnishes the lactone, completing the biosynthesis of bakkenolide A (16), which was first isolated from the flower buds of $P$. japonicus along with four other bakkenolides (B, C, D, and E) (18).

\section{Biological activity of bakkenolide A}

It was shown in previous studies that bakkenolide A exhibits selective cytotoxic activity towards cell lines derived from human carcinomas (Hep2 and HeLa) vs. HeLu and cytotoxicity against Ehrlich carcinoma (19-21). The structure and cytotoxic activity relationship of sesquiterpene lactones was investigated on numerous tumor models (21). $\alpha$-Methylene- $\gamma$-lactone moiety with exomethylene group on the lactone is essential for cytotoxicity. However, the most immediate and direct factor responsible for cytotoxicity was the $\mathrm{O}=\mathrm{C}-\mathrm{C}=\mathrm{CH}_{2}$ system (with or without lactone or cyclopentenone). The neuroprotective activity of bakkenolides from $P$. tricholobus Franch. was assayed with primary cultured neurons exposed to oxygen-glucose deprivation and oxidative insults and their antioxidant activity was investigated using cell-free bioassays (22). The obtained results showed that all the compounds exhibited notable neuroprotective and antioxidant activities. Biological activity of bakkenolide A against a variety of agricultural pests was also investigated (21). It exhibited moderate to excellent protectant activities against adult beetles ( $\mathrm{Si}$ tophilus granarius, Tribolium confusum) and larvae (Trogoderma granarium, Tribolium confusum) (23). Bakkenolide A also showed high biological activity as an antifeedant and as a larval growth inhibitor towards the variegated cutworm Peridroma saucia (24). 


\section{Major volatiles from P. hybridus}

The main compound in the essential oil from P. hybridus leaves (5) was bisabola-2,10-diene-1-one isomer. Other major compounds were dehydrofukinone, bisabola-2,10-diene-1-one derivative, germacrene $\mathrm{D}$, phytol, trans- $\beta$-caryophyllene and eremophilene. Dehydrofukinone, also known as dihydrokaranone, is an eremophilane-type sesquiterpenoid and possesses moderate peripheral and mild central analgesic activity (25) and induces sedation and anesthesia by modulation of GABAa receptors (26). P. hybridus flower stem essential oil (5) contained tricosane, bisabola-2,10-diene-1-one derivative, $\beta$-eudesmol, fukinanolid, albene, bisabola-2,10-diene-1-one and 6,10,14-trimethylpentadecan-2-one. Tricosane from $P$. japonicus flower stalk essential oil was reported previously (27). As observed in our recent study (5), P. hybridus rhizome essential oil contained bisabola-2,10-diene-1-one derivative, fukinanolid, eremophilene, hinesol, aromadendrene, $\beta$-eudesmol and bisabola-2,10-diene1-one isomer. Fukinanolid, eremophilene and albene were also previously reported as major volatiles in the rhizomes of $P$. hybridus (28). Similarly to our research, the amount of bakkenolide A was found to be significantly lower in P. hybridus buds compared to P. albus buds (19). Distinct separation of the two species was confirmed (5) by principal component analysis (PCA) and hierarchical cluster analysis (HCA). Fukinanolid, eremophilene and $\beta$-caryophyllene were also identified in the essential oils from $P$. japonicus buds and/or flower stalks and other plant parts $(27,29-32)$. Like in our previous study (5), $\beta$-caryophyllene was found dominant in leaf essential oils $(27,31)$. Larger amounts of bakkenolide A were found in the essential oils of $P$. japonicus flower stems compared to its leaves, and eremophilene was found to be typical for the root essential oil (27). This was also confirmed by our results (5) on the abundance of eremophilane structures in the rhizomes and fukinanolide in the flower stems of P. albus. More sesquiterpenes were previously found in Czech P. hybridus rhizomes, including petasin, isopetasin, $S$-petasin, furanopetasin, furanoeremophilone, eremophilenolide, petasitolide A and B, S-petasitolide A and B (33).

The most abundant compounds present in essential oils from the aerial parts of $G$. cordifolia, G. meridionalis, and G. punctata were oct-1-en-3-ol, 6-(1,5-dimethylhex-4-enyl)3-methylcyclohex-2-enone and bakkenolide A (6). Oct-1-en-3-ol is a secondary metabolite produced naturally by the enzymatic breakdown of linoleic acid; it inhibits the mycelial growth of Penicillium expansum PP497A (34). Terpenes were major compounds in the majority of Globularia oils, whereas aliphatic alcohols predominated only in one sample of $G$. cordifolia oil. Moreover, terpenes were mostly represented by oxygenated sesquiterpenes, followed by oxygenated monoterpenes and by a phytol isomer (diterpenoid). The results of multivariate statistical analyses (PCA and HCA) analyses suggested that the composition of Globularia essential oils could be useful for the evaluation of their phylogenetic relationships. Eleven compounds found in investigated samples (6) were also detected previously in two Algerian G. alypum L. samples (35). Hexadecanoic acid was the major constituent in both G. alypum samples, similarly as in one G. punctata sample included in our study (6). Moreover, oct-1-en-3-ol was present in high amounts in one G. alypum sample (35), which was also comparable to our previous results (6). According to other studies, oct-1-en-3-ol and/or eugenol were recognized as major volatiles of G. alypum $(36,37)$.

\section{CONCLUSIONS}

The present short review emphasizes the specific phytochemical composition of the essential oils of two Petasites and three Globularia species from Croatia. The determined 
phytochemical composition and distribution of sesquiterpenes with an eremophilane skeleton can be useful for chemotaxonomy of these species. According to the available data on observed activities of the major constituents present in investigated essential oils, their potential biological activities should be further investigated taking into account the possible synergy or antagonism among oil compounds.

\section{REFERENCES}

1. P. M. Dewick, Medicinal Natural Products, A Biosynthetic Approach, $2^{\text {nd }}$ ed., John Wiley \& Sons, Chichester 2002.

2. F. Rohdich, S. Hecht, A. Bacher and W. Eisenreich, Deoxyxylulose phosphate pathway of isoprenoid biosynthesis. Discovery and function of ispDEFGH genes and their cognate enzymes, Pure Appl. Chem. 75 (2003) 393-405; https://doi.org/10.1351/pac200375020393

3. F. Bakkali, S. Averbeck, D. Averbeck and M. Idaomar, Biological effects of essential oils - A review, Food Chem. Toxicol. 46 (2008) 446-475; https://doi.org/10.1016/j.fct.2007.09.106

4. K. H. C. Başer and G. Buchbauer, Handbook of Essential Oils, Science, Technology and Applications, $2^{\text {nd }}$ ed., CRC Press, Boca Raton 2016.

5. M. Friščić, I. Jerković, Z. Marijanović, S. Dragović, K. Hazler Pilepić and Ž. Maleš, Essential oil composition of different plant parts from Croatian Petasites albus (L.) Gaertn. and P. hybridus (L.) G.Gaertn., B.Mey. \& Scherb. (Asteraceae), Chem. Biodiversity 2019 (in press, doi: 10.1002/ cbdv.201800531)

6. M. Crkvenčić, S. Dudaš, I. Jerković, Z. Marijanović, D. Poljuha and K. Hazler Pilepić, Essential oil composition of three Globularia species, Chem. Biodiversity 13 (2016) 219-223; https://doi.org/10.1002/ cbdv.201500062

7. B. M. Fraga, Natural sesquiterpenoids, Nat. Prod. Rep. 24 (2007) 1350-1381; https://doi.org/10.1039/ B806216C

8. P. Claeson, R. Andersson and G. Samuelsson, T-Cadinol: A pharmacologically active constituent of scented myrrh: Introductory pharmacological characterization and high field ${ }^{1} \mathrm{H}$ - and ${ }^{13} \mathrm{C}-\mathrm{NMR}$ data, Planta Med. 57 (1991) 352-356; https://doi.org/10.1055/s-2006-960116

9. H.-T. Chang, Y.-H. Cheng, C.-L. Wu, S.-T. Chang, T.-T. Chang and Y.-C. Su, Antifungal activity of essential oil and its constituents from Calocedrus macrolepis var. formosana Florin leaf against plant pathogenic fungi, Biores. Technol. 99 (2008) 6266-6270; https://doi.org/10.1016/j.biortech.2007.12.005

10. S.-T. Chang, P.-F. Chen, S.-Y. Wang and H.-H Wu, Antimite activity of essential oils and their constituents from Taiwania cryptomerioides, J. Med. Entomol. 38 (2001) 455-457; https://doi. org/10.1603/0022-2585-38.3.455

11. G. R. Jamieson, E. H. Reid, B. P. Turner and A. T. Jamieson, Bakkenolide-A. Its distribution in Petasites species and cytotoxic properties, Phytochemistry 15 (1976) 1713-1715; https://doi. org/10.1016/S0031-9422(00)97462-4

12. P. Siegenthaler and M. Neuenschwander, Analytic investigations of sesquiterpenes of Petasites albus (L.) Gaertn, Pharm. Acta Helv. 71 (1996) 345-353; https://doi.org/10.1016/S0031-6865(96)00040-4

13. L. Novotný, V. Herout and F. Šorm, Plant substances. XVII. Constituents of Petasites albus (L.) Gaertn. rhizomes, Collect. Czechoslov. Chem. Commun. 27 (1962) 1400-1403; https://doi.org/10.1135/ cccc19621400

14. A. Srikrishna, T. J. Reddy, S. Nagaraju and J. A. Sattigeri, A stereoselective total synthesis of bakkenolide-A (fukinanolide), Tetrahedron Lett. 35 (1994) 7841-7844; https://doi.org/10.1016/00404039(94)80132-0 
15. K. Hayashi, H. Nakamura and H. Mitsuhashi, Synthesis of bakkenolide A, Chem. Pharm. Bull. 21 (1973) 2806-2807; https://doi.org/10.1248/cpb.21.2806

16. J. E. Payne, The Total Synthesis of ( \pm )-Bakkenolide-A, master thesis, The University of Calgary, Calgary 1999.

17. K. Naya, I. Takagi, Y. Kawaguchi, Y. Asada, Y. Hirose and N. Shinoda, The structure of fukinone, a constituent of Petasites japonicus maxim, Tetrahedron 24 (1968) 5871-5879; https://doi.org/10.1016/ S0040-4020(01)96317-3

18. K. Shirahata, T. Kato, Y. Kitahara and N. Abe, Constituents of genus petasites-IV : Bakkenolide-A, a sesquiterpene of novel carbon skeleton, Tetrahedron 25 (1969) 3179-3191; https://doi.org/10.1016/ S0040-4020(01)82850-7

19. G. R. Jamieson, E. H. Reid, B. P. Turner and A. T. Jamieson, Bakkenolide-A. Its distribution in Petasites species and cytotoxic properties, Phytochemistry 15 (1976) 1713-1715; https://doi.org/10.1016/ S0031-9422(00)97462-4

20. K. Kano, K. Hayashi and H. Mitsuhashi, Syntheses of steroids having a bakkenolide-type spiro-

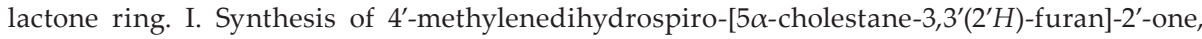
Chem. Pharm. Bull. 30 (1982) 1198-1203; https://doi.org/10.1248/cpb.30.1198

21. A. K. Picman, Biological activities of sesquiterpene lactones, Biochem. Syst. Ecol. 14 (1986) 255-281; https://doi.org/10.1016/0305-1978(86)90101-8

22. Y.-L. Wang, R.-P. Li, M.-L. Guo, G. Zhang, N. Zhang and Y.-L. Ma, Bakkenolides from Petasites tricholobus and their neuroprotective effects related to antioxidant activities, Planta Med. 75 (2009) 230-235; https://doi.org/10.1055/s-0028-1088377

23. J. Nawrot, E. Bloszyk, J. Harmatha, L. Novotný and B. Drozdz, Action of antifeedants of plant origin on beetles infesting store products, Acta Entomol. Bohemoslov. 83 (1986) 327-335.

24. J. Nawrot, O. Koul, M. B. Isman and J. Harmatha, Naturally occurring antifeedants: Effects on two polyphagous lepidopterans, J. Appl. Entomol. 112 (1991) 194-201; https://doi. org/10.1111/j.1439-0418.1991.tb01046.x

25. M. Dekić, N. Radulović, N. Stojanović and M. Mladenović, Analgesic activity of dehydrofukinone, a sesquiterpene ketone from Senecio nemorensis L. (Asteraceae), Facta Universitatis 16 (2018) 119.

26. Q. I. Garlet, L. da Costa Pires, L. H. Milanesi, J. R. Marafiga, B. Baldisserotto, C. F. Mello and B. M. Heinzmann, (+)-Dehydrofukinone modulates membrane potential and delays seizure onset by GABAa receptor-mediated mechanism in mice, Toxicol. Appl. Pharmacol. 332 (2017) 52-63; https:// doi.org/10.1016/j.taap.2017.07.010

27. M. Miyazawa, A. Teranishi and Y. Ishikawa, Components of the essential oil from Petasites japonicus, Flavour Fragr. J. 18 (2003) 231-233; https://doi.org/10.1002/ffj.1203

28. Y. Saritas, S. H. von Reuss and W. A. König, Sesquiterpene constituents in Petasites hybridus, Phytochemistry 9 (2002) 795-803; https://doi.org/10.1016/S0031-9422(01)00489-7

29. M. Sawamura, M.-S. L. Kim, K.-I. Shichiri, T. Tsuji and K. Machida, Volatile constituents of Japanese and Korean Udo (Aralia cordata Thunb.) and butterbur (Petasites japonicas Miq.), Kochi Daigaku Gakujutsu Kenkyu Hokoku, Nogaku 38 (1989) 49-60.

30. H. Shibata and S. Shimizu, Three chemovars of Petasites japonicus Maxim., Agric. Biol. Chem. 42 (1978) 1427-1428; https://doi.org/10.1080/00021369.1978.10863176

31. M. Kikuchi, Studies on the constituents of the flower stalk of Petasites japonicus Maxim. VII. on the components of the volatile oil, Yakugaku Zasshi. 93 (1973) 123-126; https://doi.org/10.1248/ yakushi1947.93.1_123

32. T. Kurihara and M. Kikuchi, Studies on the constituents of the flower stalk of Petasites japonicus Maxim. VI. On the components of the volatile oil, Yakugaku Zasshi 92 (1972) 635-638; https://doi. org/10.1248/yakushi1947.92.5_635 
33. L. Novotný, J. Jizba, V. Herout and F. Šorm, Plant Substances. XVI. The constituents of coltsfoot rhizomes (Petasites officinalis Moench), Collect. Czechoslov. Chem. Commun. 27 (1962) 1393-1399; https://doi.org/10.1135/cccc19621393

34. D. O. Okull, R. B. Beelman and H. Gourama, Antifungal activity of 10-oxo-trans-8-decenoic acid and 1-octen-3-ol against Penicillium expansum in potato dextrose agar medium, J. Food Prot. 66 (2003) 1503-1505.

35. M. Ramdani, T. Lograda, A. Ounoughi, P. Chalard, G. Figueredo, H. Laidoudi and M. ELKolli, Chemical composition, antimicrobial activity and chromosome number of Globularia alipum from Algeria, Int. J. Curr. Microbiol. Appl. Sci. 3 (2014) 306-318.

36. J. Llusiá, J. Peñuelas, G. A. Alessio and M. Estiarte, Seasonal contrasting changes of foliar concentrations of terpenes and other volatile organic compounds in four dominant species of a Mediterranean shrubland submitted to a field experimental drought and warming, Physiol. Plantarum 127 (2006) 632-649; https://doi.org/10.1111/j.1399-3054.2006.00693.x

37. B. Barhouchi, S. Aouadi and A. Abdi, Determination of eugenol and its derivative isoeugenol in Globularia alypum using solvent system extraction and comparative study of their antioxidant activities with various oxidation conditions, J. Chem. Pharm. Res. 6 (2014) 776-784. 